\title{
Comparative Studies on the Constituents of Ophiopogonis Tuber and Its Congeners. VII. Studies on the Homoisoflavonoids of the Subterranean Part of Ophiopogon japonicus Ker-GawLER cv. Nanus. (1) ${ }^{1)}$
}

\author{
Takayuki Asano, Tetsuya Murayama, Yasuaki Hirai, and Junzo ShojI* \\ School of Pharmaceutical Sciences, Showa University, Hatanodai, Shinagawa-ku, Tokyo 142, Japan. \\ Received May 29, 1992
}

\begin{abstract}
Six known homoisoflavonoidal compounds $(1-6)$ and three new homoisoflavonoidal compounds (7-9) were isolated from the ether-soluble fraction of the subterranean part of Ophiopogon japonicus Ker-Gawler cv. Nanus. Among them, 1, 2, 3, 4, 5 and 6 were identified as methylophiopogonone $A$ (1), ophiopogonone $A(2)$, methylophiopogonanone A (3), ophiopogonanone A (4), JE-III (5) and desmethylisoophiopogonone B (6), respectively. The structures of compounds 7 -9 were elucidated as $5,7,2^{\prime}$-trihydroxy-6-methyl-3-(3',4'-methylenedioxybenzyl)chromone $(7), 5,7,2^{\prime}$-trihydroxy-8-

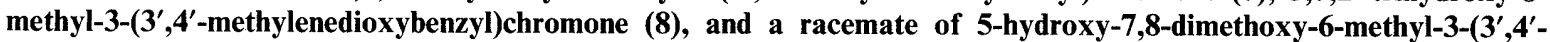
dihydroxybenzyl)chroman-4-one (9), respectively.
\end{abstract}

Keywords Ophiopogonis Tuber; Ophiopogon japonicus cv. Nanus; homoisoflavonoid; Liliaceae; homoisoflavone; homoisoflavanone

In a preceding paper, ${ }^{2)}$ the chemical constituents of the subterranean part of Liriope spicata var. prolifera and $L$. muscari (Liliaceae) were reported. The present paper deals with the isolation and structure elucidation of homoisoflavonoidal compounds of the subterranean part of Ophiopogon japonicus KeR-GAWLER cv. Nanus.

The subterranean part of $O$. japonicus KER-GAwLER cv. Nanus were extracted with hot methanol, and the methanol extract was treated by the method described in the Experimental section. Six homoisoflavonoidal compounds, 1-6 and three new homoisoflavonoidal compounds, 7-9 were isolated. Based on the infrared (IR), ultraviolet (UV), ${ }^{13} \mathrm{C}$-nuclear magnetic resonance $\left({ }^{13} \mathrm{C}\right.$-NMR $),{ }^{1} \mathrm{H}$-NMR and circular dichroism (CD) spectra of compounds 1, 2, 3, 4,5 and 6 , the compound structures were deduced to be methylophiopogonone A [5,7-dihydroxy-6,8-dimethyl-3$\left(3^{\prime}, 4^{\prime}\right.$-methylenedioxybenzyl)chromone $](1),{ }^{3)}$ ophiopogonone A [5,7-dihydroxy-6-methyl-3-(3',4'-methylenedioxybenzyl)chromone (2) ${ }^{4}$ methylophiopogonanone A [5,7-dihydroxy-6,8-dimethyl-(3R)-(3',4'-methylenedioxybenzyl)chroman-4-one] (3), ${ }^{3)}$ ophiopogonanone A [5,7-dihydroxy6-methyl-(3R)-(3',4'-methylenedioxybenzyl)chroman-4one $\quad(4),{ }^{4}$ JE-III [5,7-dihydroxy-3-(4'-hydroxybenzyl)-6methylchromone] $(5)^{5)}$ and desmethylisoophiopogonone B [5,7-dihydroxy-3-(4'-hydroxybenzyl)-8-methylchromone] (6) ${ }^{4)}$ respectively. Finally, the compounds were identified by direct comparison with an authentic sample by a mixed fusion.

Compounds 7, 8 and 9 were positive in ferric chloride reaction, and based on UV and IR spectrometric studies, compounds 7 and $\mathbf{8}$ were believed to be homoisoflavone derivatives, while compound $\mathbf{9}$ was assumed to be a homoisoflavanone derivative. The molecular weight and the number of carbon atoms of each compound were determined by mass spectrometry (MS) and by ${ }^{13} \mathrm{C}-\mathrm{NMR}$ spectrometry.

Compounds 7 and 8 showed the presence of hydroxyl, $\alpha, \beta$-unsaturated carbonyl at $1660 \mathrm{~cm}^{-1}$ and a methylenedioxy group at $924 \mathrm{~cm}^{-1}$ in IR spectra, and the MS of compounds 7 and $\mathbf{8}$ showed the same molecular ion peak $\left(\mathrm{M}^{+}\right)$at $m / z 342$, respectively. ${ }^{13} \mathrm{C}-\mathrm{NMR}$ spectra suggested that both compounds were composed of eighteen carbon atoms. The ${ }^{1} \mathrm{H}$-NMR spectra of compounds $\mathbf{7}$ and $\mathbf{8}$ showed the presence of three hydroxyl groups at $\delta 12.72,9.69,8.17$ and at $\delta 12.37,9.62,8.18$, one aromatic methyl group at $\delta 2.05\left(6-\mathrm{CH}_{3}\right)$ and at $\delta 2.16\left(8-\mathrm{CH}_{3}\right)$, one methylenedioxy group at $\delta 5.91$ and at $\delta 5.91$, one olefinic proton of C-2 at $\delta 8.08(\mathrm{H}-2)$ and at $\delta 8.05(\mathrm{H}-2)$, one methylene proton of C-9 at $\delta 3.69$ and at $\delta 3.70$ and two ortho-coupled aromatic protons at $\delta 6.37\left(1 \mathrm{H}, \mathrm{d}, J=7.9 \mathrm{~Hz}, \mathrm{H}-5^{\prime}\right), 6.76(1 \mathrm{H}, \mathrm{d}$, $\left.J=7.9 \mathrm{~Hz}, \mathrm{H}-6^{\prime}\right)$ and at $\delta 6.38\left(1 \mathrm{H}, \mathrm{d}, J=8.3 \mathrm{~Hz}, \mathrm{H}-5^{\prime}\right)$, $6.77\left(1 \mathrm{H}, \mathrm{d}, J=8.3 \mathrm{~Hz}, \mathrm{H}-6^{\prime}\right)$, respectively. Consequently, compounds 7 and 8 were suggested to be the positional isomers of homoisoflavone, whose one aromatic methyl group is located at either C- 6 or C-8 of A ring. Based on the chemical shifts of the aromatic proton and aromatic methyl group, ${ }^{5)}$ the structures of compounds $\mathbf{7}$ and $\mathbf{8}$ were established as 5,7,2'-trihydroxy-6-methyl-3- $\left(3^{\prime}, 4^{\prime}\right.$-methylenedioxybenzyl)chromone (7) and 5,7,2'-trihydroxy-8methyl-3-(3',4'-methylenedioxybenzyl)chromone (8), respectively.

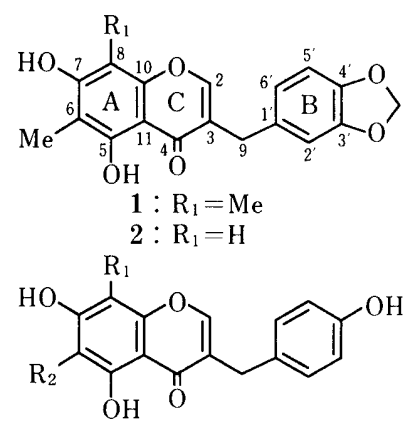

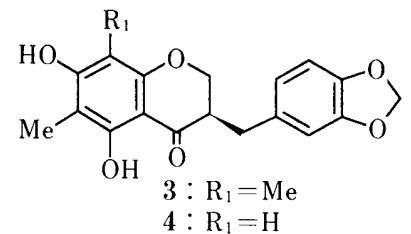

4: $\mathrm{R}_{1}=\mathrm{H}$
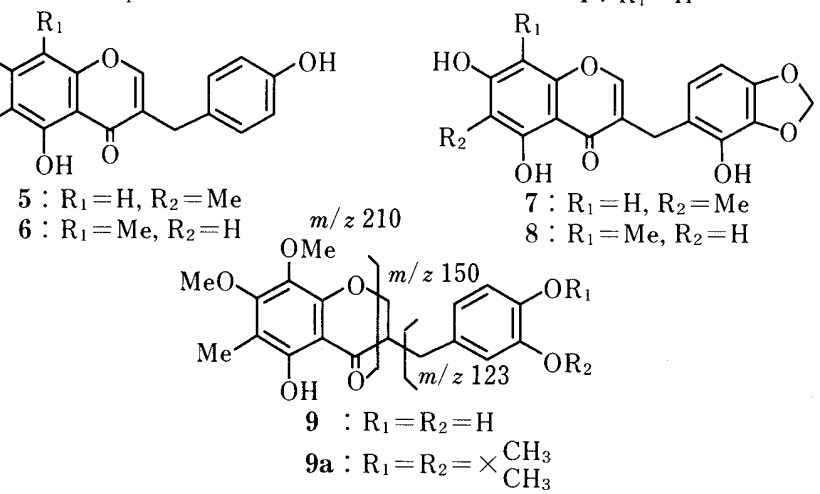

Chart 1 
The IR spectrum of compound 9 showed the hydroxyl and carbonyl absorption bands, while the MS of 9 showed the molecular ion peak $\left(\mathrm{M}^{+}\right)$at $m / z$ 360, which was suggested to have nineteen carbon atoms by ${ }^{13} \mathrm{C}-\mathrm{NMR}$ spectrum. The ${ }^{1} \mathrm{H}-\mathrm{NMR}$ and ${ }^{1} \mathrm{H}-{ }^{1} \mathrm{H}$ correlation spectroscopy (COSY) spectra of 9 showed benzylmethylene proton signals at $\delta 2.65$ and 3.21 , methylene proton signals at $\delta 4.25$ and 4.42 , methine proton signal at $\delta 3.09$, ABX type aromatic protons of $\mathrm{B}$ ring at $\delta 6.32,6.44$ and 6.96 , one aromatic methyl proton signal at $\delta 1.98$, three hydroxyl proton signals at $\delta 8.19,8.51$ and 12.12 and two methoxy proton signals at $\delta 3.75$ and 4.00 .

MS of compound 9 showed a dihydroxytropyrium fragment peak due to the B-ring at $\mathrm{m} / z 150$ and 123 and two fragment peaks due to the A-ring at $\mathrm{m} / \mathrm{z} 211$ and 210 occurred by the retro Diels-Alder cleavage reaction. Based on the MS of 9, this compound has one hydroxyl, one aromatic methyl and two methoxyl groups in A-ring and two hydroxyl groups in B-ring. Three partial structures of B ring, 3,4-dihydroxy, 2,5-dihydroxy and 2,4-dihydroxy patterns, were suggested and the substitution pattern of B-ring was finally proved by chemical reaction to form a isopropylidene ketal ring with $p$-toluenesulfonic acid and dimethoxypropane in benzene. On the other hand, the substitution pattern of the A ring of compound 9 was determined as follows. It was pointed out that the signal of C-5 carbon atom is generally shifted to the high field by about $1.5 \mathrm{ppm}$ with the addition of deuteromethanol. ${ }^{6}$ ) Based on this report, the carbon signal at $\delta 157 \mathrm{ppm}$ of ${ }^{13} \mathrm{C}-\mathrm{NMR}$ spectrum of compound 9 was assigned to $\mathrm{C}-5$ carbon atom. The long range ${ }^{13} \mathrm{C}-{ }^{1} \mathrm{H}$ COSY spectrum of compound 9 revealed cross peaks due to ${ }^{3} J_{\mathrm{C}-\mathrm{H}}\left(\mathrm{C}-5 / 6-\mathrm{CH}_{3}\right.$, $\left.\mathrm{C}-7 / 6-\mathrm{CH}_{3}\right)$ to confirm the substitution pattern of $\mathrm{A}$ ring as shown in Chart 1. Furthermore, the CD spectrum of 9 did not show any Cotton effect between 250 and $400 \mathrm{~nm}^{7)}$

Consequently, the structure of 9 was concluded to be a racemate of 5-hydroxy-7,8-dimethoxy-6-methyl-3-( $3^{\prime}, 4^{\prime}$-dihydroxybenzyl)chroman-4-one.
In this paper we have described a study on the homoisoflavonoidal constituents of Ophiopogon japonicus KER-Gawler cv. Nanus and the isolation of six known compounds $(\mathbf{1}, \mathbf{2}, \mathbf{3}, \mathbf{4}, \mathbf{5}$, and $\mathbf{6})$ and three new compounds (7, 8 and 9). Among the former, compounds 1, 3, 4, 5 and $\mathbf{6}$ were isolated from Ophiopogonis tuber and $\mathbf{2}$ was isolated from $O$. jaburan (KUNTH) LODD. Further investigation on glycosidic constituents of this plant will be reported in a later paper.

\section{Experimental}

The instruments used to obtain physical data and experimental conditions for thin layer chromatography were the same as described earlier. $\left.{ }^{2}\right)$

Extraction and Isolation The fresh subterranean part of Ophiopogon japonicus KeR-Gawler cv. Nanus $(36.6 \mathrm{~kg})$ was crushed and extracted with hot $\mathrm{MeOH}(301 \times 3)$. The extract was combined and evaporated to dryness in vacuo. The residue $(1.36 \mathrm{~kg})$ was dissolved in water and extracted with ether to afford ether-soluble fraction $(159.0 \mathrm{~g})$. The aqueous layer was extracted with $1-\mathrm{BuOH}$ saturated with water, and $\mathrm{BuOH}$-soluble fraction was concentrated in vacuo to afford a brown residue $(310.0 \mathrm{~g})$. The ether soluble fraction was subjected to column chromatography on Sephadex LH-20 with $\mathrm{CHCl}_{3}-\mathrm{MeOH}-\mathrm{H}_{2} \mathrm{O}(7: 3: 0.4, \mathrm{v} / \mathrm{v})$ to afford six fractions (frs. A-F). Fraction D was subjected to column chromatography on silicic acid with benzene-acetone $(9: 1, v / v)$ to give five fractions (frs. D-1-D-5). Fraction D-1 was subjected to column chromatography on silicic acid with benzene and the eluate was purified by recrystallization to give compounds $1(115 \mathrm{mg}), 3(1100 \mathrm{mg})$ and $9(90 \mathrm{mg})$. Fraction D-2 was subjected to column chromatography on silicic acid with hexane-acetone $(8: 2, \mathrm{v} / \mathrm{v})$ and each eluate was recrystallized to afford compounds $2(90 \mathrm{mg}), 4(43 \mathrm{mg})$ and $5(90 \mathrm{mg})$. Fraction D-5 was subjected to column chromatography on silicic acid with $\mathrm{CHCl}_{3}-\mathrm{MeOH}-\mathrm{H}_{2} \mathrm{O}$ $(7: 3: 0.4, \mathrm{v} / \mathrm{v})$ to give five fractions (frs. D-5-1-D-5-5). Fraction D-5-2 was subjected to HPLC (column; Fuji-Gel Chromatorex-ODS, $5 \mu$ m, i.d. $20 \times 250 \mathrm{~mm}$, solvent; $50 \% \mathrm{MeCN})$ to give compounds $6(40 \mathrm{mg}), 7(385 \mathrm{mg})$ and 8 (50 $\mathrm{mg})$.

Properties Compound 1: Pale yellow needles (from $\mathrm{MeOH}$ ), mp $208-210^{\circ} \mathrm{C}$. IR $v_{\max }^{\mathrm{KBr}} \mathrm{cm}^{-1}: 3520,1700,1620,900$. UV $\lambda_{\max }^{\mathrm{MeOH}} \mathrm{nm}(\log \varepsilon)$ : 292 (3.99), 266 (3.95). ${ }^{13} \mathrm{C}-\mathrm{NMR}$ : see Table I. EIMS $m / z$ : $340\left(\mathrm{M}^{+}\right), 181$, 135.

Compound 2: Pale yellow needles (from $\mathrm{MeOH}$ ), mp 199-201 ${ }^{\circ} \mathrm{C}$. IR $v_{\max }^{\mathrm{KBr}} \mathrm{cm}^{-1}: 3300,1660,1640,940$. UV $\lambda_{\max }^{\mathrm{MeOH}} \mathrm{nm}(\log \varepsilon): 294$ (3.98), 262 (3.97). ${ }^{13} \mathrm{C}-\mathrm{NMR}$ : see Table I. EIMS $m / z$ : $326\left(\mathrm{M}^{+}\right), 167,135$.

Compound 3: Colorless needles (from $\mathrm{MeOH}$ ), mp 165-166 ${ }^{\circ} \mathrm{C},[\alpha]_{\mathrm{D}}^{23}$

TABLE I. ${ }^{13}$ C-NMR Chemical Shifts of Homoisoflavonoidal Compounds

\begin{tabular}{|c|c|c|c|c|c|c|c|c|c|}
\hline Carbon & 1 & 2 & 3 & 4 & 5 & 6 & 7 & 8 & 9 \\
\hline 2 & 152.6 & 153.8 & 68.8 & 68.3 & 153.5 & 155.1 & 154.3 & 154.6 & 70.0 \\
\hline 3 & $120.7^{a)}$ & $121.1^{c)}$ & 44.8 & 44.9 & 121.4 & 121.1 & 122.0 & $121.3^{e)}$ & 45.9 \\
\hline 4 & 180.6 & 180.3 & 197.6 & 197.2 & 181.4 & 181.0 & 181.0 & 181.4 & 200.3 \\
\hline 5 & 159.2 & 161.8 & 161.9 & 160.5 & 161.8 & 161.3 & 162.3 & 162.0 & 157.0 \\
\hline 6 & 103.7 & 103.5 & 102.8 & 100.4 & 103.4 & 97.6 & 104.0 & 98.3 & $103.9^{f)}$ \\
\hline 7 & 154.0 & 155.0 & 156.8 & 164.0 & $155.1^{d)}$ & 155.1 & 155.6 & 155.3 & 160.0 \\
\hline 8 & 101.1 & 92.3 & 101.7 & 93.4 & 92.2 & 101.2 & 92.8 & 101.8 & 132.9 \\
\hline 9 & 29.2 & 29.3 & 31.1 & 29.2 & 28.6 & 28.5 & 24.4 & 24.2 & 29.6 \\
\hline 10 & 155.0 & 158.0 & 158.1 & 159.7 & 158.0 & 158.3 & 158.7 & 159.0 & 152.8 \\
\hline 11 & 106.4 & 106.3 & 100.6 & 102.8 & 106.2 & 103.7 & 107.0 & 104.2 & $104.1^{f)}$ \\
\hline 6-Me & $7.48^{b)}$ & 6.9 & 7.1 & 6.9 & 6.8 & - & 7.5 & - & 7.6 \\
\hline $8-\mathrm{Me}$ & $7.51^{b)}$ & - & 7.5 & - & - & 7.8 & - & 7.3 & - \\
\hline $1^{\prime}$ & 132.6 & 132.6 & 131.4 & 131.4 & 128.7 & 128.6 & 121.3 & $120.8^{e)}$ & 131.9 \\
\hline $2^{\prime}$ & 108.5 & 108.6 & 108.7 & 108.6 & 128.9 & 128.9 & 147.0 & 147.0 & 111.0 \\
\hline $3^{\prime}$ & 146.6 & 146.7 & 146.8 & 146.8 & 114.5 & 114.5 & 135.0 & 134.9 & $156.0^{g)}$ \\
\hline $4^{\prime}$ & 145.0 & 145.1 & 145.2 & 145.2 & $155.0^{d)}$ & 155.1 & 138.9 & 138.7 & $155.2^{g)}$ \\
\hline $5^{\prime}$ & 107.5 & 107.6 & 107.6 & 107.6 & 114.5 & 114.5 & 100.2 & 100.1 & 107.8 \\
\hline $6^{\prime}$ & $120.9^{a)}$ & $120.9^{c)}$ & 121.5 & 121.5 & 128.9 & 128.9 & 121.4 & 122.2 & 116.1 \\
\hline $\mathrm{O}-\mathrm{CH}_{2}-\mathrm{O}$ & 100.2 & 100.2 & 100.2 & 100.3 & - & - & 100.7 & 100.7 & - \\
\hline $\mathrm{OMe}$ & - & - & - & - & - & - & - & - & 60.9 \\
\hline $\mathrm{OMe}$ & - & - & - & - & - & - & - & - & 61.4 \\
\hline
\end{tabular}


$-72^{\circ}\left(c=1.00, \mathrm{CHCl}_{3}\right) . \mathrm{IR} v_{\max }^{\mathrm{KBr}} \mathrm{cm}^{-1}: 3420,1640,1630,940 . \mathrm{UV} \lambda_{\max }^{\mathrm{MeOH}} \mathrm{nm}$ ( $\log \varepsilon$ ): 296 (4.00), $214(3.86),{ }^{13} \mathrm{C}-\mathrm{NMR}$ : see Table I. EIMS $\mathrm{m} / \mathrm{z}: 342$ $\left(\mathrm{M}^{+}\right), 135 . \mathrm{CD}\left(c=5.85 \times 10^{-5}, \mathrm{MeOH}\right)[\theta]_{2}(\mathrm{~nm}):+3400(315),-12000$ (295) (negative maximum), $+5100(270)$.

Compound 4: Colorless needles (from $\mathrm{MeOH}$ ), mp 174-175 ${ }^{\circ} \mathrm{C}$, $[\alpha]_{\mathrm{D}}^{23}-63^{\circ}\left(c=1.00, \mathrm{CHCl}_{3}\right)$. IR $v_{\max }^{\mathrm{KBr}} \mathrm{cm}^{-1}: 3420,1640,1600,920$. UV $\lambda_{\max }^{\mathrm{MeOH}} \mathrm{nm}(\log \varepsilon): 294(3.98), 213(3.84),{ }^{13} \mathrm{C}-\mathrm{NMR}$ : see Table I. EIMS $\mathrm{m} / \mathrm{z}$ : $328\left(\mathrm{M}^{+}\right), 135 . \mathrm{CD}\left(c=6.11 \times 10^{-5}, \mathrm{MeOH}\right)[\theta]_{\lambda}(\mathrm{nm}):+3300(315)$, -13000 (295) (negative maximum), $+4900(270)$.

Compound 5: Pale yellow needles (from $\mathrm{MeOH}$ ), mp $250-252^{\circ} \mathrm{C}$. IR $v_{\max }^{\mathrm{KBr}} \mathrm{cm}^{-1}: 3400,1660,1627 . \mathrm{UV} \lambda_{\max }^{\mathrm{MeOH}} \mathrm{nm}(\log \varepsilon): 294$ (3.94), 262 (3.89). ${ }^{13} \mathrm{C}-\mathrm{NMR}$ : see Table I. EIMS $m / z: 298\left(\mathrm{M}^{+}\right), 167$.

Compound 6: Pale yellow needles (from $\mathrm{MeOH}$ ), mp 203-205 ${ }^{\circ} \mathrm{C}$. IR $v_{\max }^{\mathrm{KBr}} \mathrm{cm}^{-1}: 3400,1660,1627 . \mathrm{UV} \lambda_{\max }^{\mathrm{MeOH}} \mathrm{nm}(\log \varepsilon): 299$ (4.01), 260 (3.95). ${ }^{13}$ C-NMR: see Table I. EIMS $m / z$ : $298\left(\mathbf{M}^{+}\right), 167,107$.

Compound 7: Pale yellow needles (from $\mathrm{MeOH}), \mathrm{mp} 275-276^{\circ} \mathrm{C}(\mathrm{dec}$.) IR $v_{\max }^{\mathrm{KBr}} \mathrm{cm}^{-1}: 3400,1660,1625,924$. UV $\lambda_{\max }^{\mathrm{MeOH}} \mathrm{nm}(\log \varepsilon): 299(4.01), 260$ (3.95). ${ }^{1} \mathrm{H}-\mathrm{NMR}\left(\mathrm{CDCl}_{3}\right) \delta: 12.72(1 \mathrm{H}, \mathrm{s}, 5-\mathrm{OH}), 9.69\left(1 \mathrm{H}, \mathrm{s}, 2^{\prime}-\mathrm{OH}\right)$, $8.17(1 \mathrm{H}, \mathrm{s}, 7-\mathrm{OH}), 8.08(1 \mathrm{H}, \mathrm{s}, \mathrm{H}-2), 6.76\left(1 \mathrm{H}, \mathrm{d}, J=7.9 \mathrm{~Hz}, \mathrm{H}-6^{\prime}\right), 6.48$ $(1 \mathrm{H}, \mathrm{s}, \mathrm{H}-8), 6.37\left(1 \mathrm{H}, \mathrm{d}, J=7.9 \mathrm{~Hz}, \mathrm{H}-5^{\prime}\right), 5.91\left(2 \mathrm{H}, \mathrm{s},-\mathrm{O}-\mathrm{CH}_{2}-\mathrm{O}-\right)$, $3.69(2 \mathrm{H}, \mathrm{s}, \mathrm{H}-9), 2.05\left(3 \mathrm{H}, \mathrm{s}, 6-\mathrm{CH}_{3}\right) .{ }^{13} \mathrm{C}-\mathrm{NMR}$ : see Table I. EIMS $m / z$ : $342\left(\mathrm{M}^{+}\right): 167,151,107$. Anal. Calcd for $\mathrm{C}_{18} \mathrm{H}_{14} \mathrm{O}_{7}: \mathrm{C}, 63.16 ; \mathrm{H}, 4.12$. Found: C, 62.88; H, 4.09 .

Compound 8: Pale yellow needles (from $\mathrm{MeOH}$ ), mp 226-227 ${ }^{\circ} \mathrm{C}$. IR

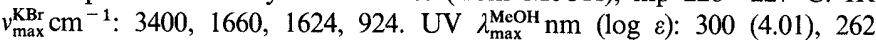
(3.96). ${ }^{1} \mathrm{H}-\mathrm{NMR}\left(\mathrm{CDCl}_{3}\right) \delta: 12.37(1 \mathrm{H}, \mathrm{s}, 5-\mathrm{OH}), 9.62\left(1 \mathrm{H}, \mathrm{s}, 2^{\prime}-\mathrm{OH}\right)$, $8.18(1 \mathrm{H}, \mathrm{s}, 7-\mathrm{OH}), 8.05(1 \mathrm{H}, \mathrm{s}, \mathrm{H}-2), 6.77\left(1 \mathrm{H}, \mathrm{d}, J=8.3 \mathrm{~Hz}, \mathrm{H}-6^{\prime}\right), 6.38$ $\left(1 \mathrm{H}, \mathrm{d}, J=8.3 \mathrm{~Hz}, \mathrm{H}-5^{\prime}\right), 6.36(1 \mathrm{H}, \mathrm{s}, \mathrm{H}-6), 5.91\left(2 \mathrm{H}, \mathrm{s},-\mathrm{O}-\mathrm{CH}_{2}-\mathrm{O}\right)$, $3.70(2 \mathrm{H}, \mathrm{s}, \mathrm{H}-9), 2.16\left(3 \mathrm{H}, \mathrm{s}, 8-\mathrm{CH}_{3}\right) \cdot{ }^{13} \mathrm{C}-\mathrm{NMR}$ : see Table I. EIMS $\mathrm{m} / \mathrm{z}$ : $342\left(\mathrm{M}^{+}\right), 167,151$, 107. Anal. Calcd for $\mathrm{C}_{18} \mathrm{H}_{14} \mathrm{O}_{7}: \mathrm{C}, 63.16 ; \mathrm{H}, 4.12$. Found: C, 63.34; H 4.07 .

Compound 9: Yellow syrup, $[\alpha]_{\mathrm{D}}^{23} \pm 0^{\circ}\left(c=0.50, \mathrm{CHCl}_{3}\right)$. IR $v_{\max }^{\mathrm{KBr}} \mathrm{cm}^{-1}$ : $3420,1620 . \mathrm{UV} \lambda_{\max }^{\mathrm{MeOH}} \mathrm{nm}(\log \varepsilon): 286(4.01), 216(3.86) .{ }^{1} \mathrm{H}-\mathrm{NMR}\left(\mathrm{CDCl}_{3}\right)$ $\delta: 12.12(1 \mathrm{H}, \mathrm{s}, 5-\mathrm{OH}), 8.51,8.19\left(\right.$ each $1 \mathrm{H}, \mathrm{s}, 3^{\prime}-\mathrm{OH}$ or $\left.4^{\prime}-\mathrm{OH}\right), 6.96(1 \mathrm{H}$, d, $\left.J=7.9 \mathrm{~Hz}, \mathrm{H}-5^{\prime}\right), 6.44\left(1 \mathrm{H}, \mathrm{d}, J=2.0 \mathrm{~Hz}, \mathrm{H}-2^{\prime}\right), 6.32(1 \mathrm{H}, \mathrm{dd}, J=7.9$, $\left.2.0 \mathrm{~Hz}, \mathrm{H}-6^{\prime}\right), 4.42(1 \mathrm{H}, \mathrm{dd}, J=12.3,4.1 \mathrm{~Hz}, \mathrm{H}-2 \alpha), 4.25(1 \mathrm{H}, \mathrm{dd}, J=12.3$, $8.2 \mathrm{~Hz}, \mathrm{H}-2 \beta), 4.00\left(3 \mathrm{H}, \mathrm{s}, 7-\mathrm{OCH}_{3}\right), 3.75\left(3 \mathrm{H}, \mathrm{s}, 8-\mathrm{OCH}_{3}\right), 3.21(1 \mathrm{H}, \mathrm{dd}$,
$J=13.3,4.8 \mathrm{~Hz}, \mathrm{H}-9 \alpha), 3.09(1 \mathrm{H}, \mathrm{m}, \mathrm{H}-3), 2.65(1 \mathrm{H}, \mathrm{dd}, J=13.3,9.6 \mathrm{~Hz}$, $\mathrm{H}-9 \beta), 1.98\left(3 \mathrm{H}, \mathrm{s}, 6-\mathrm{CH}_{3}\right) .{ }^{13} \mathrm{C}-\mathrm{NMR}$ : see Table I. EIMS $m / z: 360\left(\mathrm{M}^{+}\right)$, $211,210,150,123$. HRMS $m / z: 360.1257\left[\mathrm{M}^{+}\right]$(Calcd for $\mathrm{C}_{19} \mathrm{H}_{20} \mathrm{O}_{7}$ : 360.1209 ).

Isopropylidene Derivative of 9 (9a) A mixture of compound $9(10 \mathrm{mg})$, 4-dimethoxypropane $(0.5 \mathrm{ml})$ and a catalytic amount of $p$-toluenesulfonic acid in dry benzene was allowed to stand at room temperature for $30 \mathrm{~min}$. After neutralization with sat. $\mathrm{NaHCO}_{3}$ solution the resulting mixture was extracted with ether. The organic layer was washed with water, dried over anhydrous $\mathrm{Na}_{2} \mathrm{SO}_{4}$ and evaporated. The residue was subjected to column chromatography on Sephadex LH-20 (eluted by $\mathrm{MeOH}$ ) to afforded compound $9 \mathrm{a}(3.0 \mathrm{mg})$.

Compound 9a: Colorless syrup. ${ }^{1} \mathrm{H}-\mathrm{NMR}\left(\mathrm{CDCl}_{3}\right) \delta: 11.80(1 \mathrm{H}, \mathrm{s}$, $5-\mathrm{OH}), 3.97\left(3 \mathrm{H}, \mathrm{s}, 7-\mathrm{OCH}_{3}\right), 3.75\left(3 \mathrm{H}, \mathrm{s}, 8-\mathrm{OCH}_{3}\right), 2.02\left(3 \mathrm{H}, \mathrm{s}, 6-\mathrm{CH}_{3}\right)$, 1.21 (6H, s, gem-dimethyl).

Acknowledgements The authors are grateful to Kyoto Herbal Garden, Central Research Division, Takeda Chemical Industries, Ltd., for supplying plant material. This work was supported in part by a grant from the Uehara Medicinal Foundation. Thanks are also due to the staff of the Analytical Laboratory of this school for elemental analysis, MS and NMR spectral measurement.

\section{References}

1) A part of this work was presented at the 110th Annual Meeting of the Pharmaceutical Society of Japan, Sapporo, August 1990.

2) B. Y. Yu, Y. Hirai, J. Shoji, and G. J. Xu, Chem. Pharm. Bull., 38 1931 (1990).

3) A. Tada, R. Kasai, T. Saitoh, and J. Shoji, Chem. Pharm. Bull., 28, $1477(1980)$

4) A. Tada, R. Kasai, T. Saitoh, and J. Shoji, Chem. Pharm. Bull., 28, 2039 (1980)

5) Y. Watanabe, S. Sanada, Y. Ida, and J. Shoji, Chem. Pharm. Bull., 33, 5358 (1985).

6) Y. Terui and K. Tori, Abstracts of Papers, 17th Symposium Papers on NMR, Tokyo, 1978, p. 37.

7) M. Namikoshi and T. Saitoh, Chem. Pharm. Bull., 35, 3597 (1987). 